\title{
SERVICE LEVEL AGREEMENT IN OPTICAL NETWORKS
}

\author{
Michel Du-Pond(1), Olivier Audouin(3), Bela Berde(3), Belkacem \\ Daheb(1), Wissam Fawaz(2), Guy Pujolle(1), M. Vigoureux(3) \\ (1) University of Paris 6, LIP6 Lab, 8 rue du capitaine Scott, 75015, Paris \\ (2) University of Paris 13, L2TI, ISEP, LIP6 \\ (3) ALCATEL, Route de Nozay, 91460 Marcoussis, France
}

\begin{abstract}
This article proposes a Service Level Agreement applied to the optical domain (O-SLA), which is expected to be the near and long term network technology thanks, among other things, to the great bandwidth capacity offered by optical devices. After an exposition of the rationale behind an optical SLA, parameters which could enter in this O-SLA, as well as their values for four classes of services, are proposed. Different client (wavelength or subwavelength) and services types (from leased wavelength to bandwidth on demand) are distinguished when necessary.
\end{abstract}

Key words: SLA, SLS, Optical Networks, QoS Classes of Service.

\section{INTRODUCTION}

In an environment of fast changing technologies and uncertain business tendencies, network operators, constructors, and organizations face new challenges to keep up with the increasingly bandwidth needs of our world. This is a major driver for the technological development of optical networks, which are foreseen in the future as data centered optical networks with reduced number of electronic elements. As these new and complex networks

The original version of this chapter was revised: The copyright line was incorrect. This has been corrected. The Erratum to this chapter is available at DOI: 10.1007/978-0-387-35703-4_21

D. Gaïti et al. (eds.), Network Control and Engineering for QoS, Security and Mobility II

(C) IFIP International Federation for Information Processing 2003 
appear, automation of configuration and management tasks needs to be done, and it is in this context that the creation of specifications and standards becomes mandatory, yielding to definitions and proposals such as the one discussed in this paper. However, in the objective to converge toward a unified network, a key feature is the capability to offer differentiated services in a single network, to accommodate the different requirements of the various clients. In addition, service differentiation is a valuable opportunity for operators to increase the income that they get from their infrastructure, by selling high added-value services and get rid of present business situation where voice traffic is still dominant for revenue in spite of its less and less weight in volume.

A Service Level Agreement (SLA) is a formal contract between a service provider and a subscriber, which contains technical and detailed specifications called Service Level Specifications (SLSs).

An SLS is a set of parameters and their values, which together define the service offered to a traffic stream in a network [1]. Until now, no standards for the contents of a SLS have been defined, but interesting proposals have been published as Internet drafts by the IETF [2].

Because optical network technologies belong to an emerging domain, until now, no Service Level Agreements have been defined that are adapted to the specific needs of optical networks. Some work has been done in defining SLAs for traditional IP networks [2][3][4], but these do not consider important issues involved in optical technologies and therefore do not meet the requirements and exigencies of the next generation optical network operators and service subscribers. This work focuses on defining these SLAs specifically adapted to the relationship between optical network operators and their diverse clients.

\section{TYPES OF CLIENTS AND TYPE OF SERVICES IN AN OPTICAL NETWORK}

From now on, in this document, the relationships defined by an O-SLA consider a service provider to be an optical carrier operator, and a service subscriber to be either an optical client or an IP or MPLS client (see figure 1).

An optical client subscribes optical network services from the optical carrier operator with a granularity that is equal to a wavelength, waveband (set of wavelengths) or a complete fiber. 


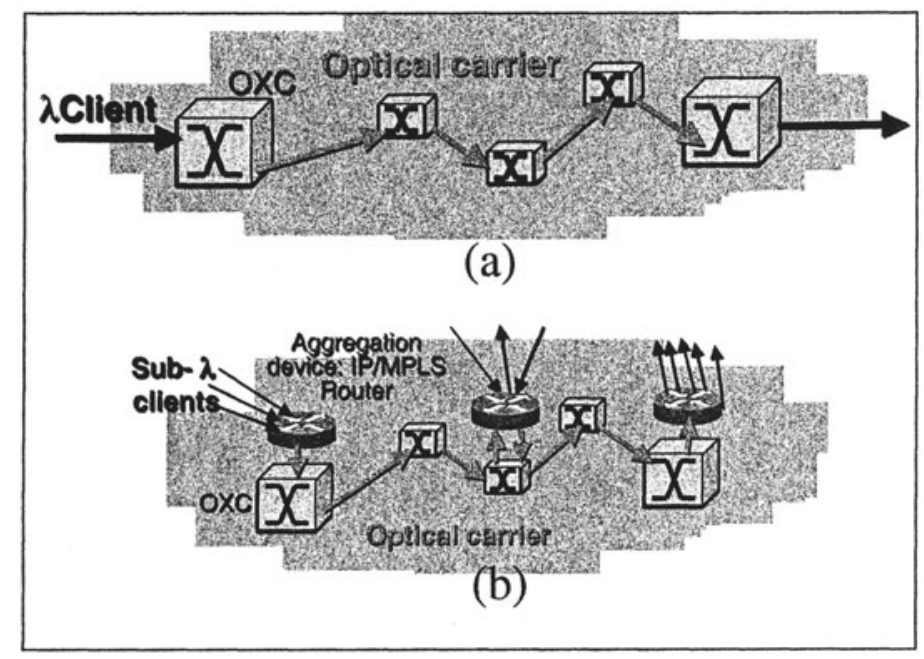

Figure 1. a) Optical client; b) IP/MPLS client

The optical client would be typically another peer optical operator whose network interacts with that of the optical carrier operator in order to provide other network services to its own clients.

The optical client can also be a large private enterprise that has its own optical equipment and subscribes optical services directly from the optical carrier operator rather than to subscribe network services from third party IP network providers.

An IP or MPLS client, within the context of this proposal, subscribes network services with a granularity which is smaller than a wavelength, and therefore its network traffic may undergo a process of grooming or aggregation provided by the optical operator's network. We suppose in this document that the aggregating device is an IP or MPLS router. Compared to previous case, it allows, as we will see, other opportunities to differentiate the service.

An example of an IP client could be an Internet Service Provider that subscribes optical network communication services from the optical carrier operator, and provides IP services to smaller clients of its own.

Another IP client could be a large enterprise that subscribes network services directly from the optical carrier operator through an IP router interface, or other under-wavelength granularity equipment such as an ATM switch or SONET/SDH device, for example. 


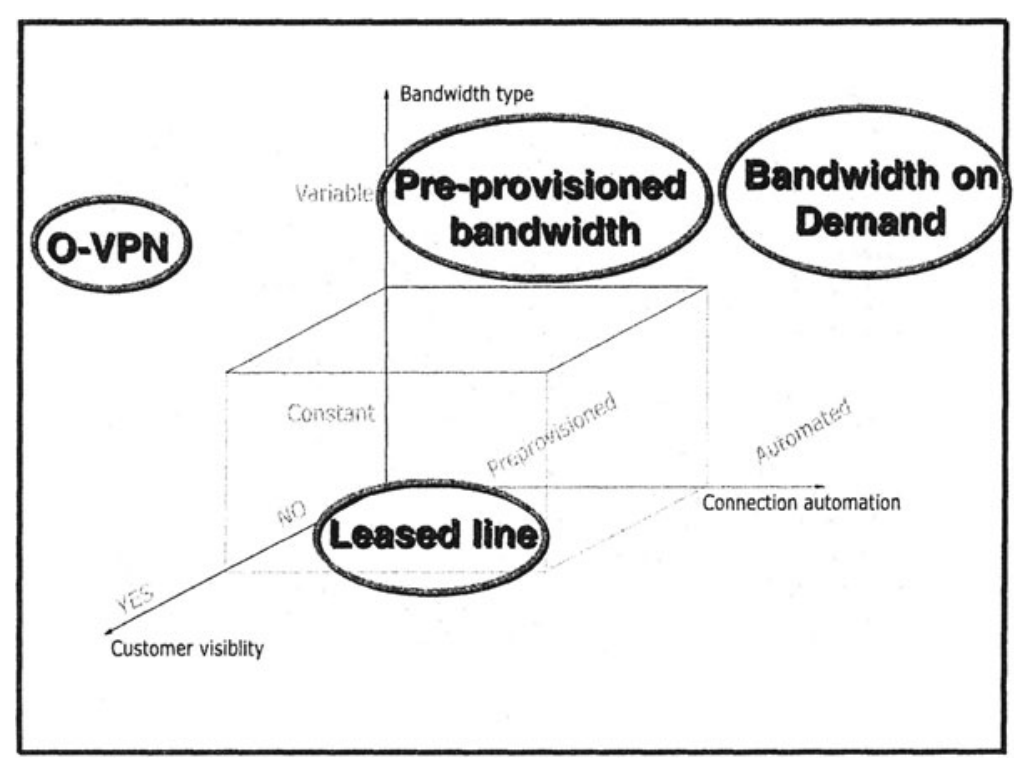

Figure 2. Typology of services

As shown in figure 2, different types of services can be envisioned in an optical network, which differs on several dimensions, that is the degree of variability of the bandwidth, the degree of automation of the connection establishment, and the degree of customer visibility on the resources which are allocated to him. We have firstly a leased-line type of service where the bandwidth is no often changed and which consequently can tolerate a low level of connection automation. In the pre-provisioned bandwidth case, we suppose that the bandwidth variations exist but that they have been scheduled in the SLA so that the carrier can easily pre-provision the resources. Bandwidth on demand case is more constraining for the operator as it requires the real time provisioning of bandwidth without previous knowledge of demand variations. A high level of connection automation is then mandatory. Finally the optical virtual private network (O-VPN) [5] is a multipoint-to-multipoint service where the customer has at least the visibility on the resource which allocated to him and possibly the opportunity to partially directly manage this resource.

\section{OPTICAL-SLA STRUCTURE AND CONTENTS}

An Optical-Service Level Agreement (O-SLA) is a formal contract between a service provider and a subscriber within the optical networks domain, which is composed of a Legal Agreement part and an Optical- 
Service Level Specification (O-SLS) that consists of technical and detailed specifications. An O-SLS is a set of parameters and their values, which together define the service offered to a traffic stream in an optical network. In this part, we are going to propose and detail the O-SLS parameters which are specific to optical networks, we will not evocate parameters which are generic and applicable to any SLS such as service boundary, service schedule and flow identifier. In addition, we propose some values for the OSLS parameters for four classes of service (from platinum to bronze [7], excluding best effort traffic for which no guarantee at all is provided).

\subsection{CONNECTION SET-UP TIME}

The Connection Set-up time specifies how long it will take for a service connection to be established once it has been negotiated and requested. Connection Set-up Time might be expressed in seconds, minutes, hours or even days, depending on the client demands and service characteristics.

This attribute is closely related to the Service Schedule, because the Connection Set-up Time determines the time that will pass between a service connection request and the actual connection activation, while the Service Schedule determines the periods during which the connection will be active as well as the duration of each period. When applicable, the periodicity of the connection is also specified in the Service Schedule.

For an operator, a longer time to establish a connection means more time to reduce resource allocated to this connection by properly optimizing routing and wavelength assignment or rearranging the network configuration, modifying if necessary other connections. As less optimization possibilities exist when the connection set-up must be rapid, this is a service that must be charged at a higher price.

Table 1 shows an example of what could be the specification of the connection set-up times for the different classes of service. We distinguished two cases for which the connection set-up time has a different meaning. For leased line and pre-provisioned bandwidth services, it represents the time between the service ordering and the service availability, a relatively long time can be tolerated, involving administrative processes and possibly some manual network configuration. For bandwidth on demand service, we deal with more real time automatic provisioning and the order of magnitude proposed for the connection time parameter is radically shorter. 
Table 1. Connection set-up times

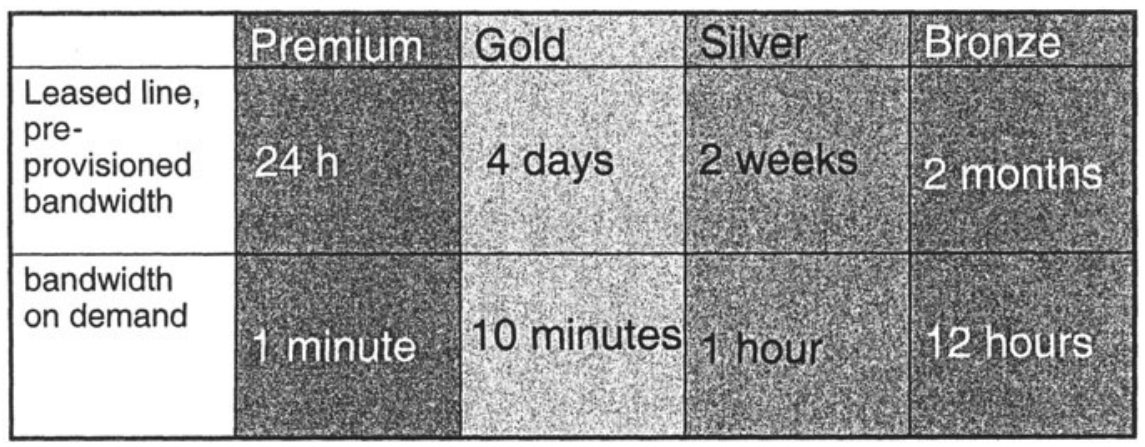

\subsection{SERVICE AVAILABILITY AND RESILIENCE}

We propose the following parameters for differentiation of service availability (this equally applies to both optical and IP/MPLS clients):

- Out-of-Service Criterion

- Service Recovery Time

- Recovery Time with Degraded Performance

- Service Mean Down Time.

The Out-of-Service Criterion controls the triggering of the resilience mechanism. It can be a fault (loss of power) or degradation (degraded BER) as some applications may tolerate degraded BER and other not.

Next, we defined two recovery times. The first one, the Service Recovery Time defines the time needed to recover the full initial SLS parameters. It can be completed with a shorter second time period during which the connection is recovered but some degradation of SLS parameters (in particular service performance guaranties of paragraph 3.4) are tolerated. Note that no particular resilience scheme (restoration or protection, $1+1$ or $\mathrm{M}: \mathrm{N}$...) is indicated in the SLS, it is a decision which pertain to the operator and which should not be made visible for the client, the only constraint for the operator being to fulfill the specified recovery times.

The Service Mean Down Time is the maximum service breakdown time allowed during a year. It can be specified in seconds or as a percentage.

Table 2 shows an example of what could be these different parameters for the four classes of services. 
Table 2. Service availability and resilience

\begin{tabular}{|c|c|c|c|c|}
\hline & Premium & Gold & Silver & Bionze \\
\hline $\begin{array}{l}\text { Out of service } \\
\text { criterion }\end{array}$ & $\begin{array}{l}\text { Degraded } \\
B E R=10\end{array}$ & $\begin{array}{l}\text { Degraded } \\
B E R=10^{-3}\end{array}$ & $\begin{array}{l}\text { Fault } \\
(L O S)\end{array}$ & $\begin{array}{l}\text { Favilf } \\
\text { ros }\end{array}$ \\
\hline $\begin{array}{l}\text { Recovery time with } \\
\text { degraded SLA }\end{array}$ & & $50 \mathrm{~ms}$ & & \\
\hline Full recovery time & $50 \mathrm{~ms}$ & $300 \mathrm{~ms}$ & & \\
\hline $\begin{array}{l}\text { Service } \\
\text { unavailability }\end{array}$ & & & & \\
\hline
\end{tabular}

\subsection{ROUTING CONSTRAINTS}

The process of routing connections within the network offers several possibilities for service differentiation.

\subsubsection{ROUTING STABILITY}

The Routing Stability determines whether optical traffic trunks can be rerouted or not, and in the case in which it is agreed by the optical operator and its clients that the traffic trunks can be rerouted, it also specifies how often this will take place.

From the client point of view, Routing Stability is another critical attribute of the O-SLS, since relevant QoS parameters such as delay, throughput, jitter, and loss can be degraded if rerouting takes place very often. Re-routing can also induce some service interruption affecting the overall service availability. In some cases, certain applications might be especially sensitive to rerouting, for clients using this kind of applications; the periodicity of rerouting should be set to be very small or null. On the contrary, for applications that are non-sensitive to rerouting periodicity, a higher periodicity might be set in exchange for lower billing of the service.

From the optical operator point view, Routing Stability is a very important issue because, when a client requests a service that involves a long duration or permanent connection that cannot be rerouted due to specific application and business characteristics, then the operator has strong constraints. One of the constrains concerns optimization of network resource allocation, because the optical operator cannot tear down the client's 
permanent connection even if overall channel utilization becomes very low, this results in a waste of bandwidth and optical resources. Also, in such case, the operator cannot do intermediate grooming to optimize network and bandwidth utilization with the connection in question. Additionally, the routing blocking probability increases due to the inability to reroute a certain connection. Given the number of constraints that such a service imposes on the optical operator's network, the optical operator can apply higher billing for this kind of service, compensating in this way for the inconveniencies of avoiding rerouting for a certain connection.

On the other hand, Routing Stability is of importance to the optical operator because it can also offer services that provide for a connection to be rerouted a definite number of times. This allows the optical operator to re optimize its network resource allocation from time to time, tear down connections in case of underutilization, perform intermediate grooming, and decrease routing blocking probabilities by providing more possible routes when calculating paths to establish new requested connections.

\subsubsection{ROUTE DIFFERENTIATION}

This attribute involves physical path differentiation, and also "Shared Risk Link Group" (SRLG as defined by the IETF [6]) path differentiation. Clients who desire to manage by themselves, protection and restoration of their connections, may request services consisting of two or more Label Switch Paths that do not belong to the same SRLG, or do not share any physical links or nodes. Clients might also demand, for security or other reasons, a service in which none of the links and/or nodes passes through a certain country or territory.

For the optical network operators, such services represent important routing constraints and, just as in the Routing Stability attribute, the routing blocking probability increases due to the necessity to use different physical paths for two or more Label Switch Paths that begin and end at the same end points of the network. This has also an important impact in network resource allocation optimization and efficient utilization. Additionally, when determining the different routes to set up connections throughout the network, these routing constraints need to be considered, this involves complicated routing tools and mechanisms. Due to these complications and constraints, the optical operator can apply higher billing for this kind of service in order to compensate for these inconveniencies as well as to increase income and build up competitive advantage derived from better service offers that apply and meet its client's needs in an optimal way. 


\subsubsection{CONFIDENTIALITY}

Confidentiality is a very important issue in all network and information services in general. Optical networks are not the exception, thus different confidentiality levels and constraints need to be defined. The Confidentiality attribute defines what kind of confidentiality level will be associated to the service subscribed in the O-SLS. In optical networks, the best way to provide a confidential connection is using a transparent connection. A lower confidentiality level, for an IP/MPLS is to avoid any grooming with other clients on a same wavelength. This can be applied to a part of the route only, in the area of the network considered as critical. This represents for the operator additional constraints impacting resource usage efficiency.

\subsubsection{DISTANCE}

This attribute represents the geographical distance between the end points of the network involved in the service defined by the O-SLS. The Distance attribute should be defined for service billing purposes only. For example, a client subscribing to a service with a connection from Paris to London will pay less than another client subscribing to the same type of service but with a connection from Paris to New York.

However, the distance attribute should not be understood as the actual length of the route allocated to the client connection, which depends on the operator network operation policy, it is consequently proprietary information not to be exposed to the client. 
Table 3: Service differentiation in routing

\begin{tabular}{|c|c|c|c|c|}
\hline & remilum & Gold & Silver & Bronze \\
\hline Routing stability & $\begin{array}{l}2 \text { times pe } \\
\text { year }\end{array}$ & $\begin{array}{l}1 \text { time per } \\
\text { month }\end{array}$ & $\begin{array}{l}\text { time pel } \\
\text { week }\end{array}$ & $\begin{array}{l}\text { No } \\
\text { limitation }\end{array}$ \\
\hline Route differentiation & $\begin{array}{l}\text { Optional } \\
\text { Fully } \\
\text { supported } \\
\text { (ink node } \\
\text { SRIG) }\end{array}$ & $\begin{array}{l}\text { Optional } \\
\text { Partially } \\
\text { supported } \\
\text { (link, node) }\end{array}$ & $\begin{array}{l}\text { Optional } \\
\text { partially } \\
\text { supported } \\
\text { (lik) }\end{array}$ & $\begin{array}{l}\text { Not } \\
\text { supported }\end{array}$ \\
\hline Confidentiality & $\begin{array}{l}\text { Optional } \\
\text { Eully } \\
\text { supported } \\
\text { (O/E } \\
\text { grooming) }\end{array}$ & $\begin{array}{l}\text { Optional } \\
\text { Partially } \\
\text { supported } \\
\text { (grooming) }\end{array}$ & $\begin{array}{l}\text { Not } \\
\text { suppoited }\end{array}$ & $\begin{array}{l}\text { Not } \\
\text { supp }\end{array}$ \\
\hline
\end{tabular}

\subsubsection{CLASSES OF SERVICE AND ROUTING CONSTRAINTS}

Table 3 shows the proposed parameters for the different classes of service. Route differentiation and confidentiality are options which are fully, partially, or not supported according to the class of service.

\subsection{SERVICE PERFORMANCE GUARANTEES}

For an IP/MPLS client the performance parameters will be those of a classical IP network, they will be impacted in particular by the priorities given to the different clients in the routers performing the aggregation. These parameters are: Delay, Jitter, Throughput and Packet loss.

For an optical client where no aggregation occurs in the optical network, performance parameter list is restricted to the throughput and the delay. The differences with the previous case is that the throughput can only have discrete values which are multiple of the bit rate granularity offered by the optical network and that the delay is impacted by the propagation distance only as no buffering occurs. Table 4 shows the values which could be allocated to these parameters for each class of service. 
Table 4: Performance guarantees

\begin{tabular}{|c|c|c|c|c|}
\hline & Premium & Gold & Silver & Bronze \\
\hline Case 1 & & & & \\
\hline Throughput & $1 \times \times \mathrm{GL}$ & $n \times x \mathrm{~Gb} / \mathrm{s}$ & $n \times 0 \mathrm{~Gb} / \mathrm{s}$ & $1 \times \times$ Cols \\
\hline Max Delay & $25 \mathrm{~ms}$ & $50 \mathrm{~ms}$ & unspecified & unspe \\
\hline Case 2 & & & & \\
\hline Throughput & Any & Any & Any & Any \\
\hline Max Delay & $35 \mathrm{~ms}$ & $100 \mathrm{~ms}$ & $500 \mathrm{~ms}$ & \\
\hline Jitter & $3 \mathrm{~ms}$ & $10 \mathrm{~ms}$ & $50 \mathrm{~ms}$ & 1. 5 \\
\hline Packet loss & $10^{9}$ & $10^{6}$ & & \\
\hline
\end{tabular}

\subsection{TRAFFIC CONFORMANCE AND EXCESS TREATMENT}

Before the traffic of the client enters the optical network, a testing of the traffic conformance characteristics must be carried out by the optical carrier operator in order to determine if the traffic conforms to what has been agreed in the O-SLS under the Traffic Conformance attribute. If the traffic test determines that the data flow is in agreement with the traffic conformance parameters defined for that data flow, then the data flow is considered to be in-profile traffic, otherwise, if there is a violation of the traffic conformance parameters previously defined, the traffic is considered to be out-of-profile traffic. The Excess Treatment attribute determines how the service provider will process excess or out-of-profile traffic. Excess traffic may be shaped or degraded. Considering that one of the key characteristics of next generation optical carrier networks that are being deployed, is the ability to provide guaranteed QoS; under normal circumstances no excess traffic should be dropped, it should be shaped or degraded. Only in the case in which accepting and processing excess traffic compromises the network's capacity to assure the QoS guaranteed to all other users at that time in the network, the excess traffic may be dropped. Typically, the regime could be shaping for premium and gold classes, and degradation for the two other classes. 


\subsubsection{IP/MPLS CLIENT CASE}

Considering the case of an IP client, the Traffic Conformance attribute describes the characteristics of the data stream identified by the Flow Identifier. The Traffic Conformance attribute contains a set of parameters that describe what the data stream should look like to get the QoS guarantees that have been indicated in the O-SLS by the Performance Parameters attribute. The following is a non-exhaustive list of potential conformance parameters [2]:

- Peak rate $\mathrm{p}$ (bits per second)

- Maximum Transmission Unit (MTU) M (bytes)

- Minimum packet size (bytes)

Excess traffic can be shaped at the entry point of the network, until it becomes in-profile traffic and is then forwarded through the network. Degradation, for an IP client, means that out-of-profile traffic will be forwarded by the routers connected to the crossconnects (see figure $2 b$ ) with inferior QoS guarantees, resulting in a degradation of logical performances.

\subsubsection{OPTICAL CLIENT CASE}

Considering now the case of an optical client, the above parameters are not relevant as we do not have access to client packets. However, same approach with physical parameters can be adopted to classify the client into in-profile or out of profile one. The following list is a non-exhaustive of potential conformance parameters:

- Wavelength drift (nm)

- Power (dBm)

- Error rate

- Chirp (GHz)

- OSNR

A "physical shaping" could be then envisioned for the out-of-physical profile signal through OEO regeneration provided by the optical carrier. In absence of such a regeneration, the physically out of profile signal will naturally undergo degradation by further propagation in the optical network. However, the relevance of such parameters depends on the way the optical client is connected to the optical network. In the likely case where the signal is systematically regenerated at the interface, they would not apply. 


\section{CONCLUSIONS}

The worldwide network domain tendency to evolve towards transparent optical networks is imminent; therefore it results of great importance and interest to contribute to the development and improvement of these emerging technologies. Part of this crucial work is being done by important groups and organizations through different research projects. This paper aims at contributing to this purpose of finding; enhancing and proposing appropriate solutions to existing problems.

Relevant steps in SLA definition have been taken by different organizations and standardization groups; and their results represent a good base to define any type of SLA. After having analyzed these projects and studied the major trends, issues and needs concerning optical networks and the technologies therein included, the O-SLA described in this paper has been proposed for the purpose of meeting the optical operators' needs and those of their clients, and to provide a guideline in that which concerns service negotiations and agreements.

An O-SLA, contracted between a client and an optical operator, have to be provisioned into the network. One solution would be to provision it by policy. In this case, the provisioning process results from the translation of the O-SLS into policy rules, First into high-level policy rules then into lowlevel (device) ones. A future work will address this issue.

\section{REFERENCES}

1. F. De Turck, S. Vanhastel, F. Vandermeulen, P. Demeester, "Design and Implementation of a Generic Connection Management and Service Level Agreement Monitoring Platform Supporting the Virtual Private Network Service", IEEExplore 2001.

2. D. Goderis, Y. T'joens, C. Jacquenet, G. Memenios, G. Pavlou, R. Egan, D. Griffin, P. Georgatos, L. Georgiadis, P. Van Heuven, "Service Level Specification Semantics and Parameters", draft-tequila-sls-01.txt, Internet Draft, June, 2001. Work in progress.

3. S. Salsano, F. Ricciato, M. Winter, G. Eichler, A. Thomas, F. Fuenfstueck, T. Ziegler, C. Brandauer, "Definition and usage of SLSs in the AQUILA consortium", draft-salsanoaquila-sls-00.txt, Internet Draft, November, 2000. Work in progress.

4. S. P. Romano, M. Esposito, G. Ventre, G. Cortese, "Service Level Agreements for Premium IP Networks", draft-cadenus-sla-00.txt, Internet Draft, November 2000. Work in progress.

5. Bela Berde, "Contribution to a Management Plane for Optical Networks operating a GMPLS Control Plane", Alcatel-CIT, Research and Innovation, NG-NSM.

6. Eric Mannie, "GMPLS Architecture", draft-ietf-ccamp-gmpls-architecture-07.txt, May 2003 Work in progress.

7. W. Zhao, D. Olshefski, H. Schulzrinne, "Internet Quality of Service: an Overview", Columbia University. 